\title{
Reflets
}

Revue ontaroise d'intervention sociale et communautaire

\section{Réponses des associations communautaires de la région de Sudbury à la crise de l'État-providence}

\section{Rachid Bagaoui}

Volume 3, numéro 1, printemps 1997

Enfance et familles en contexte d'appauvrissement

URI : https://id.erudit.org/iderudit/026153ar

DOI : https://doi.org/10.7202/026153ar

Aller au sommaire du numéro

Éditeur(s)

Reflets : Revue ontaroise d'intervention sociale et communautaire

ISSN

1203-4576 (imprimé)

1712-8498 (numérique)

Découvrir la revue

Citer cet article

Bagaoui, R. (1997). Réponses des associations communautaires de la région de Sudbury à la crise de l’État-providence. Reflets, 3(1), 102-112.

https://doi.org/10.7202/026153ar

Tous droits réservés (C) Reflets : Revue ontaroise d'intervention sociale et communautaire, 1997
Ce document est protégé par la loi sur le droit d'auteur. L'utilisation des services d’Érudit (y compris la reproduction) est assujettie à sa politique d'utilisation que vous pouvez consulter en ligne.

https://apropos.erudit.org/fr/usagers/politique-dutilisation/ 


\section{Réponses des associations communautaires de la région de Sudbury à la crise de l'État-providence}

\section{Rachid Bagaoui}

Université Laurentienne

Les associations communautaires connaissent depuis plusieurs années un regain d'intérêt de la part des chercheurs au Canada (Greg 1995; Lévesque 1995; Bélanger et Lévesque 1992; Favreau 1995; Gagnon et Klein 1991; Hamel 1991). Celles-ci rejoignent les préoccupations de plusieurs recherches de tendance internationale (Laville 1994; 1992; Vienny 1994; Defouny 1994; Vidal 1992; Whul 1991; Rodriguez 1990). En dépit de leur grande diversité, tous ces travaux convergent vers l'idée principale que le monde associatif opère actuellement un virage important dans le sens d'une intégration des exigences économiques aux objectifs sociaux et identitaires et ce, dans une démarche partenariale ainsi que locale et régionale. Certains vont même jusqu'à se demander si ces nouvelles dynamiques pourraient se révéler un modèle de développement innovateur de création d'emploi et de réponse à de nouvelles demandes sociales (Lévesque 1996).

Cet engouement s'explique par la gravité de la crise et de ses coûts sociaux. En effet, après une période de croissance et de stabilité relative des modes de régulation, nous sommes entrés, depuis les années 1970, dans une période de grands bouleversements sociaux. Cette période est marquée, entre autres, par 
une crise de l'État-providence, de l'emploi et par le déclin des grandes idéologies mobilisatrices. Le milieu associatif n'échappe pas à cette mutation. Les acteurs de ce milieu subissent les effets de la crise mais, en même temps, ils questionnent leurs stratégies d'intervention et de mobilisation ainsi que les mythes et les représentations qui les ont guidés jusqu'à maintenant. De ces questionnements, de nouvelles pratiques émergent qui tentent de répondre à la nouvelle réalité sociale.

L'intérêt pour l'analyse du milieu associatif est notoire. Il est peu marqué toutefois en Ontario francophone où l'on connait peu de choses à ce sujet, sinon que le milieu associatif est en train d'opérer un renouveau dans le discours en direction de l'économie et du partenariat (Cardinal et al., 1994). Le présent texte vise à faire le point sur la situation actuelle des associations communautaires franco-ontariennes dans la logique de la crise. Pour ce faire, le texte analyse la lecture que font les acteurs des associations de la crise de l'Etat-providence et de ses effets sur leurs organisations, ainsi que leurs réponses à cette crise. Les questions qui découlent de cette thématique se lisent comme suit. Quelles sont les difficultés auxquelles font face les intervenantes et les intervenants de ce milieu dans le contexte de la crise? Quels sont les effets de la crise sur la vie associative? Quelles sont les réponses à la crise? Quels sont les enjeux qui se dégagent de ces réponses?

Pour répondre à ces questions, nous avons entrepris depuis quelques mois une recherche à travers l'Ontario francophone ${ }^{1}$. Notre recherche, il faut le dire, n'en est qu'à ses débuts. Les résultats présentés sont donc forcément partiels. Ils représentent néanmoins une introduction à la compréhension des mutations que connaît le milieu associatif en Ontario francophone. Étant donné l'avancement de la recherche, nous présenterons dans ce texte les résultats de l'enquête menée dans la région de Sudbury auprès d'une quinzaine d'organismes oeuvrant dans le secteur de l'éducation, de l'économie, de la santé et de la culture. Au total, une quinzaine d'entrevues ont été réalisées entre avril et juin 1996. L'enquête se poursuit dans d'autres régions. 


\section{Associations communautaires franco-ontariennes et la crise}

«Outre le problème de financement qui frappe durement les associations communautaires, il y a la menace qui plane sur les acquis des francophones (dans le domaine de la santé, de l'éducation, etc.).»
Le mouvement associatif est confronté à un nombre important de problèmes dont le plus inquiétant est le financement. Cela n'est pas étonnant dans le contexte de la montée des politiques néo-libérales prônées par plusieurs provinces canadiennes dont l'Ontario. Le refrain du gouvernement conservateur en matière de finances est désormais connu: l'heure est à l'assainissement des dépenses publiques et à la rationalisation. Les mots d'ordre sont sous-traitance, privatisation, franchisage et concurrence. Ce sont là en même temps les recettes proposées pour remettre l'Ontario sur les rails. Ces politiques et les stratégies qui les animent ont de nombreuses conséquences sur les organismes étudiés. Bien qu'elles different d'un organisme à l'autre, ces conséquences se résument à quelques points majeurs.

Outre le problème de financement qui frappe durement les associations communautaires, il y a la menace qui plane sur les acquis des francophones (dans le domaine de la santé, de l'éducation, etc.). Comme groupe minoritaire, cette population sera certainement, selon les répondants, amenée tôt ou tard à se tourner vers la majorité pour répondre à ses besoins, et c'est l'assimilation qui l'attend. C'est également la disparition des services en français à brève échéance qui inquiète les intervenantes et les intervenants. L'idée que le secteur privé aura la main-mise sur ces services préoccupe plusieurs organismes et les oblige à réévaluer leurs priorités et leur stratégies.

La crise et les politiques des pouvoirs publics ont également un impact sur les conditions de travail des intervenantes et des intervenants. Les coupures dans les postes de travail les amènent à un plus grand investissement dans le travail. Ils sont contraints de faire du temps supplémentaire, de consacrer de nombreuses heures au travail à chaque journée. Étant donné le manque de ressources, ils sont obligés de s'occuper de toutes les tâches dans l'organisme. Selon plusieurs, cette situation conduit au stress, à 
des problèmes familiaux et de santé. De plus, ils ne peuvent même pas compter sur le travail des bénévoles pour alléger leur fardeau, car le bénévolat semble en perte de vitesse. Les répondants sont unanimes à l'effet que les gens ne s'intéressent plus aux affaires francophones. Surtout les jeunes qui, selon plusieurs répondants, connaissent à peine l'existence de ces organismes. Le recrutement des bénévoles est difficile car, dans un tel contexte, les responsables des organismes sont amenés à exiger beaucoup trop d'eux.

Devant cette situation morose, la démobilisation semble le lot des acteurs du milieu associatif. Les répondants semblent «fatigués de se battre». Selon eux, «les choses ne bougent pas aussi rapidement qu'ils le voudraient» et «ils se sentent seuls». Travailler dans un milieu minoritaire semble «une bataille sans fin». Plusieurs ont indiqué qu'ils auraient dû faire un métier «normal». De plus, ils semblent ne plus savoir où «donner la tête» sur le plan idéologique. Les problèmes quotidiens mobilisent davantage leur énergie. Ils n'ont plus le temps de s'impliquer dans les grands débats de sociéte. Que pensent-ils, par exemple, du pluralisme culturel, de la mondialisation? Pas grand chose, sinon que ces problèmes ne les touchent pas. Une certaine indifférence se dégage de leurs propos. Les témoignages des répondants et des répondantes laissent donc entendre que c'est l'immédiat qui les mobilise. Selon un bon nombre d'entre eux, toute vision à moyen ou à long terme est une utopie.

La situation semble donc grave. Tellement grave que le discours néo-libéral influence les attitudes des répondants. Selon un certain nombre d'entre eux, le milieu associatif doit s'adapter à la tendance en cours. En clair, cela veut dire qu'il faut "privatiser certaines activités culturelles pour compétionner avec le secteur privé, vendre le bilinguisme des Franco-Ontariens pour attirer le financement; il faut couper dans les dépenses»; il faut «mettre l'accent sur les résultats, l'efficacité». On propose également de "fusionner certains organismes», voire même de fermer ceux «qui ne sont pas rentables». Il faut «changer de priorités, de revendications pour répondre aux exigences des bailleurs de fonds». Tout cela n'est pas étonnant lorsque l'on sait que pour bon nombre de ces associations, l'État demeure le pourvoyeur 
principal. Formées autour de l'État et grâce à l'État, ces associations dépendent des subventions des pouvoirs publics et de leurs priorités pour assurer leur survie.

\section{Une communauté divisée}

Si le contexte actuel semble imposer sa logique au fonctionnement de ces organismes, pour beaucoup de répondants, ce n'est là qu'une partie du problème. Le déchirement de la population francoontarienne est un problème inquiétant. C'est une population qui, en raison de luttes internes, demeure difficile à mobiliser. La critique est également très sévère à l'endroit du leadership francoontarien. Selon des intervenantes et intervenants, celui-ci est traditionnel, encore trop axé sur l'école. Pour ces répondants et ces répondantes, ces revendications demeurent certes importantes, mais elles "occupent trop de place». Le leadership «manque de cohésion», et il est "difficile à mobiliser» car le leadership luimême est incapable de «mobiliser les ressources dont disposent les francophones».

Les problèmes que connaissent les acteurs des associations communautaires ainsi que le discours néo-libéral qui traverse leur position semblent donc réels. Mais s'en tenir à ces conclusions risque de masquer le travail de renouveau qui est en train de s'opérer au sein du milieu associatif. C'est sur ce point majeur que nous cherchons à attirer l'attention. L'hypothèse soutenue dans notre recherche est la suivante. Bien que la crise frappe durement, le mouvement associatif entre dans une période non pas de déclin ou de démobilisation mais de mutation ${ }^{2}$. Le renouvellement de l'action associative se traduit dans le discours comme sur le terrain par, entre autres, de nouvelles formes de financement et de prestation de services ainsi que de nouvelles pratiques reposant sur des exigences économiques et sociales. Notre hypothèse s'inscrit dans le prolongement des travaux développés au Canada (Favreau 1995;Bélanger et Lévesque 1994; 
Greg 1995; Thériault 1984), en France (Alvine 1994; Defourny et Monzon Campos 1992) et aux États-Unis (Vidal 1992). Ces travaux ont en commun de problématiser la question associative en terme de renouvellement, qu'il soit socio-économique, politique ou identitaire. L'ensemble de cette problématique dépasse déjà une analyse en terme de crise et tente de repérer, dans leur diversité, les réponses en voie d'émergence.

\section{Renouveau de l'action associative : la fin d'une époque}

Les associations communautaires franco-ontariennes ont beaucoup changé depuis les années 1980 (Cardinal et al. 1994). La remise en cause de l'État-providence pour des motifs non seulement de crise des finances publiques mais aussi d'exclusion et de dépendance (Lévesque 1995) vient insuffler une nouvelle vitalité au mouvement associatif. Dans chaque région, il existe un important réseau associatif, sous des appellations et des formes juridiques certes différentes, qui tente à partir de son champ d'action particulier de répondre aux besoins nouveaux et criants de la population fortement touchée par la crise. Notre recherche fait ressortir plusieurs enjeux que nous considérons comme un renouveau de l'action associative franco-ontarienne.

La liste est longue. Nous nous contentons de présenter ici les enjeux majeurs qui reviennent le plus souvent dans le discours des répondantes et des répondants.

\section{Rapport à l’État}

Bien que l'État demeure toujours une référence, les répondants s'entendent sur le fait que les Franco-Ontariens doivent cesser de se tourner vers l'État pour trouver des solutions à tous leurs problèmes. Il faut, disent-ils, «se prendre en main», «se sentir maître 
chez soi», "se développer». Cette prise en main passe, selon les répondants, par l'autonomie - par rapport aux pouvoirs publics - sur le plan du financement. On pense qu'il faut diversifier les sources de financement, par le biais de fêtes, de levées de fonds, de dons, et surtout, d'institutions financières comme les Caisses populaires. Cette autonomie doit également se déployer sur le plan local car, toujours selon les répondants et les répondantes, la communauté ontarienne francophone n'est pas homogène; il existe donc des différences fondamentales entre les régions. C'est pourquoi selon eux les stratégies des pouvoirs publics doivent tenir compte de la spécificité du territoire où s'enracine chaque organisme. Elle doit enfin se déployer en fonction de la spécificité du champ d'action des organismes. On demande à l'État, en effet, d'établir ses critères en fonction des besoins du secteur d'activité de l'organisme. Bref, les organismes sont en train de redéfinir leurs rapports à l'État. Conscients de leur dépendance vis-à-vis les pouvoirs publics, ils cherchent de nouvelles stratégies qui leur permettraient de s'approprier leur propre développement.

\section{Virage économique}

"Les répondants sont unanimes à dire que le temps où les exigences économiques étaient séparées des préoccupations sociales est révolu.»
C'est un autre terrain où les organismes tentent de se redéfinir. Cette orientation s'inscrit dans un mouvement amorcé au tournant des années 1980 alors que la plupart des associations incitaient les milieux francophones à effectuer le virage économique (Cardinal et al. 1994). Les répondants sont unanimes à dire que le temps où les exigences économiques étaient séparées des préoccupations sociales est révolu. Cette stratégie économique n'a rien de commun avec celle qui proposait, au cours des années 1980, que le virage économique se fasse par le développement de l'entrepreneurship. En fait, tout en continuant à assumer les préoccupations sociales et identitaires, les intervenantes et intervenants du milieu associatif semblent vouloir assumer aussi une partie des contraintes associées au marché: on propose que l'on s'oriente vers la création d'emplois, la formation, la production 
des biens et services. Selon les répondants, la question économique n'est plus vue comme une renonciation aux objectifs sociaux. Bref, il faut voir le développement des Franco-Ontariens comme un tout, dans sa globalité.

\section{Partenariat}

"Un autre thème qui domine le discours des intervenantes et des intervenants des associations communautaires est celui du partenariat.»
Un autre thème qui domine le discours des intervenantes et des intervenants des associations communautaires est celui du partenariat. Ce partenariat se déploie à trois niveaux. Les répondants proposent, premièrement, de développer des partenariats avec les pouvoirs publics. Il s'agit de participer aux définitions des priorités, aux critères de financement et à l'élaboration des projets. Pour tirer la meilleure partie de la situation actuelle, les répondants proposent, deuxièmement, un partenariat avec les institutions (et les groupes) anglophones. Comment? En procédant "par contrat», "par projet», «au sein d'une structure locale de développement et en fonction de certaines priorités». Mais y a-t-il un risque d'assimilation pour les Franco-Ontariens? Les acteurs semblent conscients des conséquences de ce genre de partenariat. Ils s'entendent, cependant, pour dire que la solution à ce problème réside dans le fait que les francophones doivent se laisser guider dans leur démarche, non seulement par des impératifs économiques, mais aussi identitaires (langue et culture). Autrement dit, en intégrant les références identitaires dans le rappport avec l'Autre, les francophones réussiraient, selon plusieurs, à contrer le pouvoir assimilateur de la majorité. Le discours porté par la plupart des répondants met enfin l'accent sur un renforcement de la solidarité entre les Franco-Ontariens. Refusant de laisser évoluer la situation actuelle, ce discours propose de rendre la solidarité - qui existe de façon informelle - plus formelle, avec une structure reconnue. Les éléments qui ressortent de cette stratégie sont les suivants : les Franco-Ontariens doivent se regrouper entre eux, se connaitre davantage, échanger leurs idées, développer des projets, mettre les ressources en commun, partager les coûts, etc. 
Les leçons qui se dégagent de cette brève présentation sont que le discours du milieu associatif semble s'éloigner du discours misérabiliste des années 1970. Il se caractérise par son ouverture à de nouveaux thèmes tels que le partenariat avec l'État et avec la majorité, la prise en charge et le développement économique. Cette ouverture à des thèmes nouveaux n'a pas éclipsé la question identitaire et les objectifs sociaux que poursuivent les organismes franco-ontariens. Bien entendu, certains thèmes ne sont pas nouveaux (Farmer 1984). Néanmoins, le contexte actuel a rendu ces thèmes incontournables. Quelle interprétation peut-on donner à ces pratiques? Il est tentant de conclure que l'action associative est en crise puisqu'elle rompt avec le militantisme des années 1970. Mais telle n'est pas notre position. Pour nous, l'action associative, malgré les obstacles auxquels elle doit faire face, semble en mutation - mutation allant dans le sens d'une intégration des exigences actuelles dans l'action et le discours. Cette mutation pourrait se révéler une voie prometteuse compte tenu des problèmes criants de la population franco-ontarienne. Toutes ces transformations posent cependant de sérieuses questions qui demeurent pour l'instant sans réponse. Ce discours est-il généralisé à l'ensemble des associations communautaires? Comment ce discours se traduit-il dans la réalité? Existe-t-il des initiatives qui vont dans le sens des tranformations en cours? Plus important encore: ces initiatives signifient-elles un véritable renouveau ou un simple palliatif à la crise de l'État-providence? Trouverontelles de l'appui de la part des pouvoirs publics et du milieu? Ce sont là quelques questions auxquelles notre recherche se propose de répondre dans ses prochaines étapes.

\section{Bibliographie}

BÉLANGER, P.R. et B. LÉVESQUE (1992). «Le mouvement populaire et communautaire : de la revendication au partenariat (1963-1962)», dans Le Québec en jeu, sous la dir. de Daigle et Rocher, Montréal, PUM, 713-748.

BOUCHER, J. et L. FAVREAU (1994). «Les identités à la CSN: d'une vision d'avant-garde à une position de solidarités plurielles», dans Les identités:Actes du colloque de L'ACSALF 1992, sous la dir. de Jacques Hamel et J.-Y.Thériault, Montréal, ACSALF/Méridien, 357-372. 
BRODHEAD, D., F. LAMONTAGNE et C. PEIREC (1990). L'organisme de développement local, une perspective canadienne, no 20, Ottawa, Conseil économique du Canada.

CARDINAL, L. et al. (1994). L'épanouissement des communautés de langue officielle, Ottawa, Ministère du Patrimoine canadien/Commissariat aux langues officielles.

DEFOUNY, J. (1994). Développer l'entreprise sociale, Fondation du roi Beaudoin.

DEFOURNY, J. et J. MONZON CAMPOS (1992). Économie sociale : entre économie capitaliste et économie publique/The third sector: cooperative, mutual and non profit organizations. Bruxelles, De Boeck University.

FARMER, D. (1984). Dynamique interne et développement communautaire. Une étude comparative, Thèse de maîrise, Sociologie, Ottawa.

FAVREAU,L. (1989). Mouvement populaire et intervention communautaire: continuités et ruptures, Montréal, Éd. du Fleuve/CFP.

FAVREAU, L. (1995). Relancer l'économie et l'emploi par le développement économique communautaire, Cahiers de CRISES/CRÉEOM (rapport de recherche).

FAVREAU, L. et B. LÉVESQUE (1995). Repenser le développement communautaire et l'économie sociale à la faveur de la crise de l'emploi et de l'État-providence, Cahiers CRISES, no 9504.

FAVREAU, L. et W. NINACS (1992). «Le développement économique local communautaire au Québec», Coopératives et Développement, vol. 23, no 2, 115-123.

FONTAN, J.-M. (1990). «Les corporations de développement économique communautaire: une des avenues du mouvement social dans l'économique», Coopératives et Développement, vol. 21, no 2, Montréal, PUQ/HEC.

FONTAN,J.-M. (1992). «La démocratie économique communautaire», Possibles, vol. 16, no 1, hiver 1992, 53-64.

GAGNON, C. et KLEIN, J.-L. (1989). Le local en mouvement, GRIR coll. Développement régional, Chicoutimi, UQAC.

GREG, A. (1994). «Fragmentation ou vitalité? Les nouveaux réseaux associatifs dans l'Acadie du Nouveau-Brunswick», dans Pour un espace de recherche au Canada Français. Discours, objets et méthodes, sous la dir. de B. Cazabon, Ottawa, Les Presses de l'Université d'Ottawa, 94-125.

JAQUIER, C. (1992). Les quartiers américains, rêve et cauchemar, (le développement communautaire et la revitalisation des quartiers aux États-Unis), Paris, Éd. L'Harmattan.

KLEIN,J.-L et C. GAGNON (1989). Le social apprivoisé (le mouvement associatif,l'État et le développement local), Hull, Éd. Asticou.

LACHAPPELLE, R. (1990). Le mouvement communautaire à Sorel-Tracy : éléments pour une stratégie de développement, Sorel, Éd. Communautés militantes.

LAFERRIÈRE, S. (1990). «La dynamique communautaire montréalaise», Relations, no 584, octobre 1992, 239-245.

LAVILLE, J.-L. (1992). Les services de proximité en Europe, Paris, Syros/Alternatives.

LAVILLE, J.-L. (1994). Cohésion sociale et emploi, Paris, Desclée de Brouwer.

LÉVESQUE, B. (1995). Repenser l'économie pour contrer l'exclusion sociale: de l'utopie à la nécessité, Cahiers du CRISES, no 9502.

THÉRIAULT,J.-Y. (1985). La société civile ou la chimère insaisissable : essai de sociologie politique, Montréal, Québec-Amérique. 
VIDAL, A.C. (1992). Rebuilding Communities: A National Study of Urban Community Development Corporations, New York, Community Development Research Center, Graduate School of Management and Urban Policy, New School for Social Research.

VIENNY, C. (1994). L'économie sociale, Repères, no.148, Paris, Éd. La Découverte.

WHUL, S. (1991), Du chômage à l'exclusion, l'état des politiques et l'apport des expériences, Paris, Syros Alternatives.

\section{Notes}

1. Cette recherche est menée en collaboration avec Simon Laflamme, professeur au département de sociologie à l'Université Laurentienne.

2. Notre recherche s'inspire d'un schéma de travail semblable développé par Louis Favreau (1989). 